\title{
Development and Application of Smart Geogrid Embedded with Fiber Bragg Grating Sensors
}

\author{
Zheng-fang Wang, ${ }^{1}$ Jing Wang,, Qing-mei Sui,, ${ }^{1,2}$ Xun-mei Liang, \\ Lei Jia, ${ }^{1}$ Shu-cai Li, ${ }^{4}$ and Shi-de $\mathrm{Lu}^{3}$ \\ ${ }^{1}$ College of Control Science and Engineering, Shandong University, Jingshi Road 17923, Jinan 250061, China \\ ${ }^{2}$ Shandong University Suzhou Graduate School, Lingquan Street 377, Suzhou 215123, China \\ ${ }^{3}$ TAIAN Road Engineering Materials Co. Ltd., Taiwen Road, Taian 271000, China \\ ${ }^{4}$ Geotechnical Engineering Center, Shandong University, Jingshi Road 17923, Jinan 250061, China
}

Correspondence should be addressed to Jing Wang; wangjingkz@sdu.edu.cn

Received 8 October 2014; Revised 18 December 2014; Accepted 28 December 2014

Academic Editor: Fei Dai

Copyright (C) 2015 Zheng-fang Wang et al. This is an open access article distributed under the Creative Commons Attribution License, which permits unrestricted use, distribution, and reproduction in any medium, provided the original work is properly cited.

\begin{abstract}
Smart geogrids embedded with fiber Bragg grating (FBG) for reinforcement as well as measurement of geotechnical structures have been developed. After the fabricating process of the geogrids is detailed, finite element (FE) simulations are conducted to analyze the strain distribution of geogrids and the strain transfer characteristics from geogrids to fiber optic. Results indicate that FBG should be deployed in the middle of the geogrids rib to make sure that uniform strain distribution along the FBG. Also, PVC protective sleeves, which are used to protect fiber optic when integrated with geogrids, have smaller strain transfer loss than nylon sleeves. Tensile experiments are conducted to test strain measurement performance of proposed geogrids, and the results demonstrate that proposed smart geogrids have good linearity and consistency. Temperature experiments show that FBG embedded in geogrids has higher temperature sensitivity, and the temperature induced error can be compensated by an extra FBG strain-independent sensor. Furthermore, designed smart geogrids are used in a geotechnical model test to monitor strain during tunnel excavation. The strain tendency measured by smart geogrids and traditional strain sensor agree very well. The results indicate that smart geogrids embedded with FBGs can be an effective method to measure strains for geological engineering related applications.
\end{abstract}

\section{Introduction}

Geosynthetics in form of geogrids have been extensively utilized to fulfill reinforcement of geotechnical structures such as dikes, dams, railways, embankments, landfills, and slopes [1-4]. By integrating with a series of sensors, the geosynthetics are capable of sensing strains, temperature or other parameters when used for reinforcement [5]. These multifunctional or even "smart" geosynthetics can sense and react to critical mechanical deformation/load or other external stimuli in geotechnical structure, which make them promising materials in geological engineering to prevent potential disasters and ensure safe construction and operation of civil engineering.

Over the past two decades, adhered electrical resistance strain gauges had been widely used as sensing components when integrated with geogrids, and diverse methods for installing strain gauges on geogrids were investigated [69]. However, the strain gauges adhered on geogrids were susceptible to fall off, and the gauges might be affected by moisture or electromagnetic interference (EMI) when being used in harsh environments, which provided unreliable strain measurement results.

Fiber optic sensors, both distributed fiber optic sensor and quasidistributed optic fiber Bragg grating (FBG) sensor, have a series of advantages over traditional electrical sensors when integrated with Geogrids, soil nails and other geotechnical reinforcements [10-16]. Due to their fibrous nature, fiber optic sensors can be ideally processed like standard warps threads to embed within geogrids. Moreover, fiber optic sensors exhibit better long-term reliability, since they are noncorrosive, immune to EMI, waterproof, and 

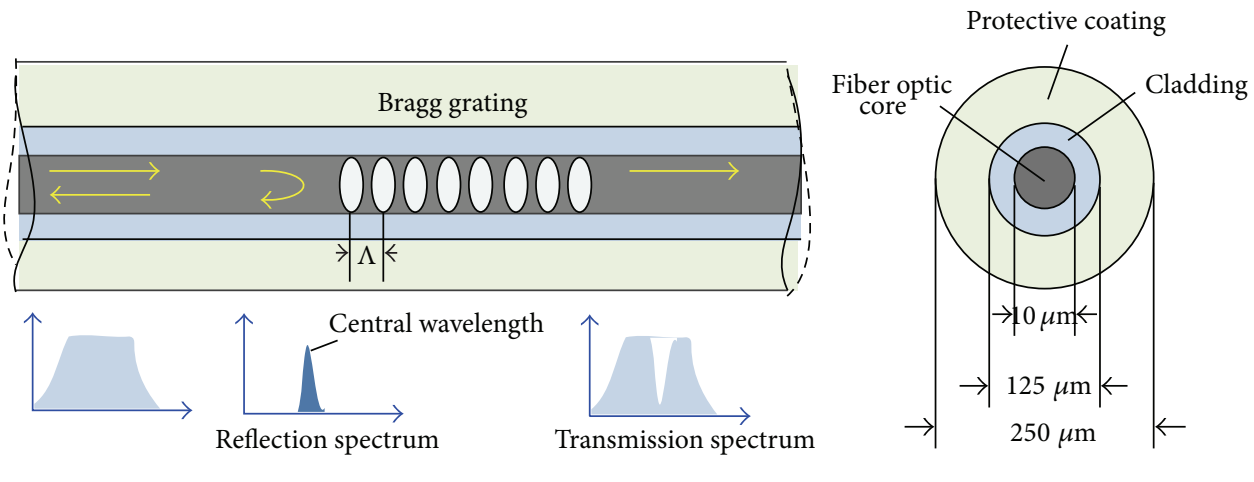

FIGURE 1: Schematic principle of fiber Bragg grating.

intrinsically safe. Additionally, fiber optic sensors based sensing system can be used for distributed/quasidistributed realtime monitoring and then delivering all sensing information through one single fiber optic cable [17-21]. Therefore, the smart geogrids integrated with fiber optic sensor have attracted considerable attention. Krebber et al. developed smart geotextiles integrated with distributed silica optic fiber (for strain of less than 1\%) and polymer optic fiber (POF) (for strain of more than $40 \%$ ), respectively, and applied them for dike and slope monitoring [22-24]. These smart geotextiles which are on the basis of distributed fiber optic sensor are ideal for geotechnical structures with large dimensions. Nevertheless, due to the limitation of its strain measurement accuracy and spatial resolution, the distributed fiber optic sensor is not applicable for some applications where accurate measurement of local strain is required (such as geotechnical model test). As a widely used fiber optic component, FBG is able to measure local strain with better accuracy and reliability compared to the distributed fiber optic sensor, it is thus necessary to investigate smart geogrids embedded with FBG for the demands of more extensive applications.

In this research, an in-depth study regarding development and application of smart geogrids embedded with fiber Bragg grating sensors is conducted. Two finite element models are built to analyze the strain distribution of FBG embedded in the geogrids and the strain transfer behavior from geogrids to FBG. Then, details of the calibration experiments, including tensile experiments and temperature experiments are reported, and the experimental results are analyzed. Finally, the designed smart geogrids are utilized in a geotechnical model test to monitor strain variation during the excavation of tunnel. The results demonstrate that proposed smart geogrids are a promising solution for reinforcement and measurement of geotechnical structure with small dimension and could be utilized in civil engineering related applications.

\section{Development of Smart Geogrids Embedded with FBG}

2.1. FBG Sensing Principle. The principle of FBG technology is shown in Figure 1. It is formed by inscribing permanent and periodic modulation of the refractive index along a short section $(<10 \mathrm{~mm})$ inside the fiber optic core [25]. This periodic refractive index demodulation, called as grating, enables FBG to reflect light with special wavelength on the basis of the Bragg law:

$$
\lambda_{B}=2 \cdot n_{\mathrm{eff}} \cdot \Lambda
$$

where $\lambda_{B}$ is the Bragg wavelength representing the wavelength at which reflection occurs, $\Lambda$ is the grating period, and $n_{\text {eff }}$ is the effective refractive index of fiber core.

The reflected Bragg wavelength $\lambda_{B}$ will shift as a function of ambient temperature $T$ and/or axial strain of fiber optic $\varepsilon$, as expressed in

$$
\Delta \lambda_{B}=\lambda_{B} \cdot\left[(\alpha+\xi) \cdot \Delta T+\left(1-P_{e}\right) \Delta \varepsilon\right]
$$

where $\Delta \lambda_{B}$ is the wavelength shift, $\alpha$ and $\xi$ are coefficient of thermal expansion and thermal-optic coefficient, respectively, and $P_{e}$, whose value is 0.22 , is valid photoelastic coefficient. Thus, $\Delta \lambda_{B}$ is proportional to axial strain $\varepsilon$ and/or temperature $T$.

2.2. Fabrication of Smart Geogrids. It is a pivotal and challenging task to integrate fiber optic into geogrids during the weaving process. The warps knitted PET geogrids are selected as the carrier of fiber optic cable due to their yarns are not easy to bend in the entire weaving process. A bunch of PET warps thread is replaced by the fiber optic cable which consist of protective sleeves and fiber optic with FBG arrays $(10 \mathrm{~mm}$ gauge length). The cable is then guided to machine with other warps thread and wove with weft threads. Double-axis warpsknitting machine utilized for fabrication of smart geogrids, needs to be operated very carefully so as to precisely control the movement of the warps threads, weft threads, and binder yarns. The junctions of warps (both with and without fiber optic cable) and weft threads are bound automatically by binder yarns with high strength.

Geogrids being knitted only are unavailable for application since their low stiffness. Also, their junctions are susceptible to slip, which may cause the embedded fiber optic to be stripped from geogrids. Thus it is essential to coat the geogrids by special polymer. Geogrids being coated have high stiffness, resistance to corrosion, and better integration with embedded fiber optic. It is thereby applicable to geotechnical 


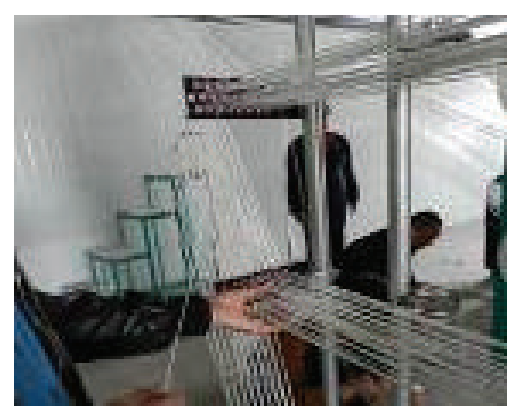

(a) Warps with fiber optic cable

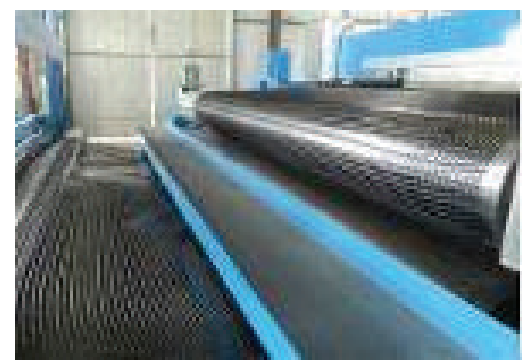

(d) Coating

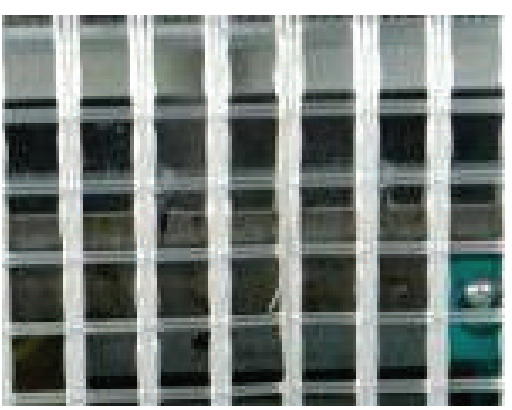

(b) Knitting

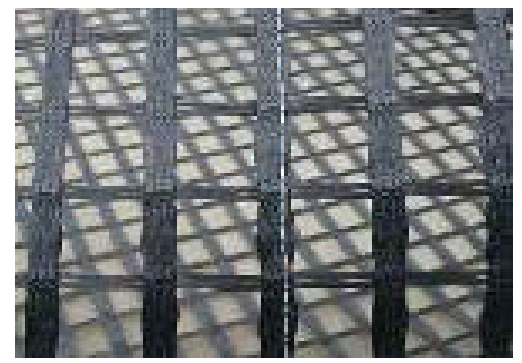

(e) Smart geogrid with FBG

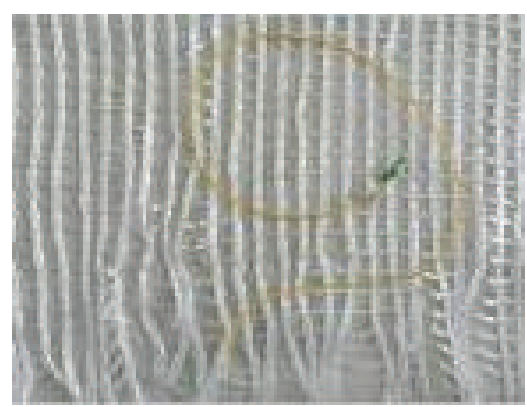

(c) Geogrid being knitted only

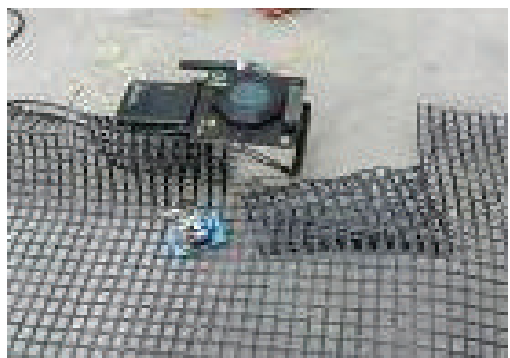

(f) Testing

FIgURE 2: Fabrication of smart geogrids.

engineering for reinforcement and measurement. Figure 2 shows the fabricating steps of warps-knitted geogrids embedded with FBG.

Three important issues need to be highlighted during the fabrication: (1) To minimize the influence of bending which is inevitable in geotechnical engineering related application, each bunch of warps threads is marked and the threadsguiding equipment is refitted to adjust the fiber optic into the centerline of geogrids' thickness direction. (2) Since the fiber optic with FBG is more fragile compared with POF and normal silica fiber optic without FBG, the tightly bonded polymer protective sleeves are designed to protect fiber optic with FBG and ensure strain transfer from geogrids to FBG. The protective sleeves can also work as a buffer to protect fiber optic when strain on the geogrids exceeds the maximum strain of the fiber optic. (3) When suffering from nonuniform strain, the spectrums of FBG will distort and cause unreliable measurement results. To solve this issue, finite element simulation is used to analyze strain distribution profile of geogrids and determine optimal location for FBG where strain profile is uniform.

\section{Simulations and Analysis}

To get reliable strain results, one important task when considering integration of fiber optics into geogrids is to ensure accurate strain transfer from ribs of geogrids to FBG. Another factor need to be noted is strain distribution profile along embedded FBG should be uniform to prevent spectrum being distorted. In this section, finite element simulations are utilized to simulate the strain distribution of geogrids with different specifications and to analyze the strain transfer characteristics from geogrids to fiber optic cable with FBG.
3.1. Strain Distribution of Geogrids under Tensile Displacement. Two finite element models of warps knitted PET geogrids with different specifications were established, as shown in Table 1. Six 3D cylinders with a diameter of $3 \mathrm{~mm}$ were set up and bonded together to simulate the six bunches of warps threads. For the weft threads, 3D cuboid models were built, and the junctions of warps and wefts threads were bonded. One end of the geogrids was fixed, and uniform tensile displacement of $3.6 \mathrm{~mm}$, which is $1 \%$ elongation $(10000 \mu \varepsilon)$, was applied on the other end of the geogrids model. The visco-elastic model was selected for calculation.

Figure 3 shows the simulated results of two geogrids (mesh size $40 * 40 \mathrm{~mm}$ and $25.4 * 25.4 \mathrm{~mm}$ ) when elongation is $1 \%$. The strain along the axis where fiber optic cable is embedded is shown in Figure 4. The strain distribution profiles of both geogrids are not uniform under tensile stress. As can be seen in Figure 4(a), strains along the two geogrids' ribs are approximately equal whereas the strain at the junction area shows a significantly decreases due to the stress concentration. The strain profiles within one rib are shown in Figure 4(b) which depicts approximate $32 \mathrm{~mm}$ in the middle section of the rib distributed evenly for geogrids $(40 * 40 \mathrm{~mm})$, while for geogrids $(25.4 * 25.4 \mathrm{~mm})$ the length is about $18 \mathrm{~mm}$. Due to the difficulty for precisely controlling the position of FBGs, geogrids $(40 * 40 \mathrm{~mm})$ are better for the integration of FBG (10 mm grating gauge) in contrast to geogrids $(25.4 * 25.4 \mathrm{~mm})$.

3.2. Analysis of Strain Transfer. To understand strain transfer behavior of smart Geogrids embedded within FBG and determine appropriate polymer materials to protect fiber optic, finite element model of geogrids $(40 * 40 \mathrm{~mm})$ was built to analyze strain transfer from geogrids' ribs to fiber 


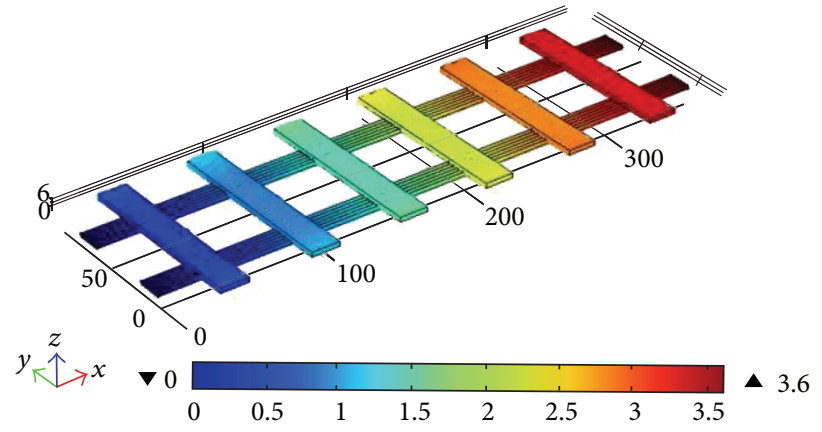

(a) Geogrids with mesh size of $40 * 40 \mathrm{~mm}$

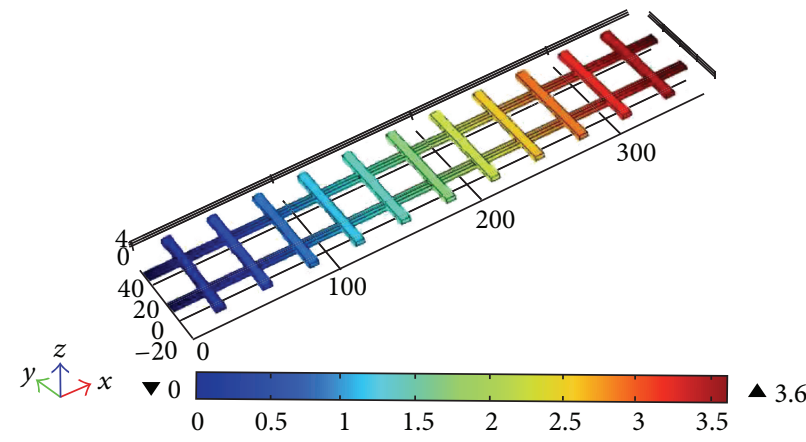

(b) Geogrids with mesh size of $25.4 * 25.4 \mathrm{~mm}$

FIGURE 3: Simulation model and displacement display.

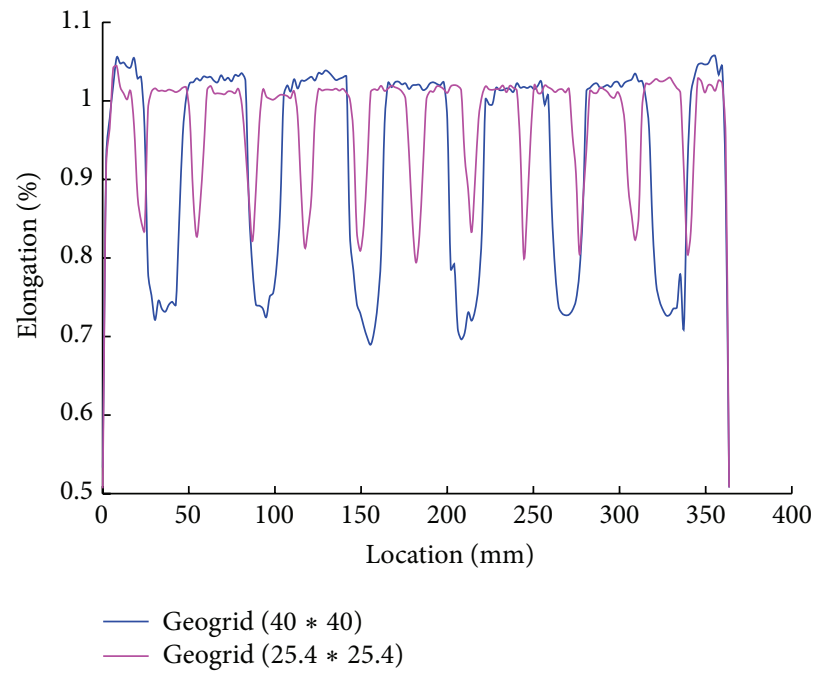

(a) Strain distribution of geogrids

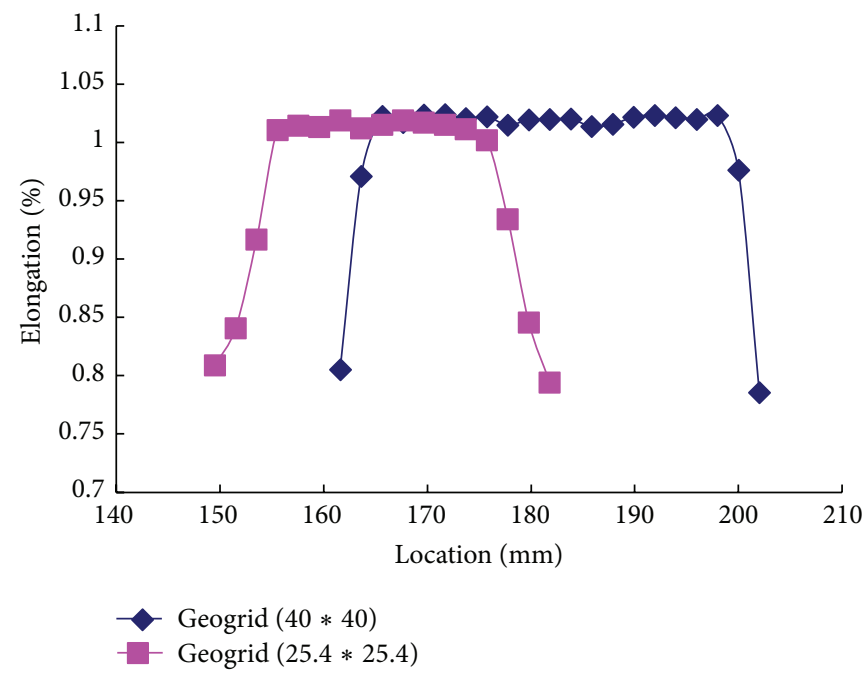

(b) Strain distribution of one rib

FIGURE 4: Strain distribution of two geogrids.

TABLE 1: Specifications of the two geogrids models.

\begin{tabular}{lcc}
\hline & G1 & G2 \\
\hline Mesh Size $(\mathrm{mm} * \mathrm{~mm})$ & $40 * 40$ & $25.4 * 25.4$ \\
Number of Warps & 6 & 3 \\
Number of Wefts & 6 & 2 \\
Length $(\mathrm{mm})$ & 360 & 360 \\
Width $(\mathrm{mm})$ & 116 & 64 \\
Thickness $(\mathrm{mm})$ & 3 & 2 \\
\hline
\end{tabular}

optic. Since the protective sleeves are tightly adhered with fiber optic to minimize strain transfer loss, and the fiber optic cable is knitted and coated with PET warps threads together, it is assumed in the simulation that interface between geogrids and protective sleeve is no-slipping and so is the interface between protective sleeve and fiber optic.

Both PVC and nylon were selected as protective sleeve in the simulation. Parameters of the two materials are shown in Table 2, the other parameters used in the simulation are
TABLE 2: Parameters used in the FE simulation.

\begin{tabular}{lccc}
\hline & PVC & Nylon & Fiber optic \\
\hline Elastic modulus $(\mathrm{GPa})$ & 3.92 & 8.3 & 73.1 \\
Poisson's ratio & 0.38 & 0.28 & 0.17 \\
Diameter $(\mathrm{mm})$ & 1 & 1 & 0.25 \\
\hline
\end{tabular}

provided by industrial collaborator (TAIAN Road Engineering Materials co. Ltd). Six parallel 3D cylinders each with a diameter of $3 \mathrm{~mm}$ were built as the model of six bunches of warps threads, and fiber optic was modeled by one $3 \mathrm{D}$ cylinder with a diameter of $0.25 \mathrm{~mm}$. Between the fiber optic and warps threads, one 3D annular model with a thickness of $0.375 \mathrm{~mm}$ was built as the protective sleeve. All the interfaces were bonded together. The fiber optic cable was placed in the middle of the six warps threads as can be shown in Figure 5.

The visco-elastic material model was applied on warps threads and its coating, while the elastic material model was selected for fiber optic and protective sleeve. The fiber optic, protective sleeve and coating were defined as free constraint, 


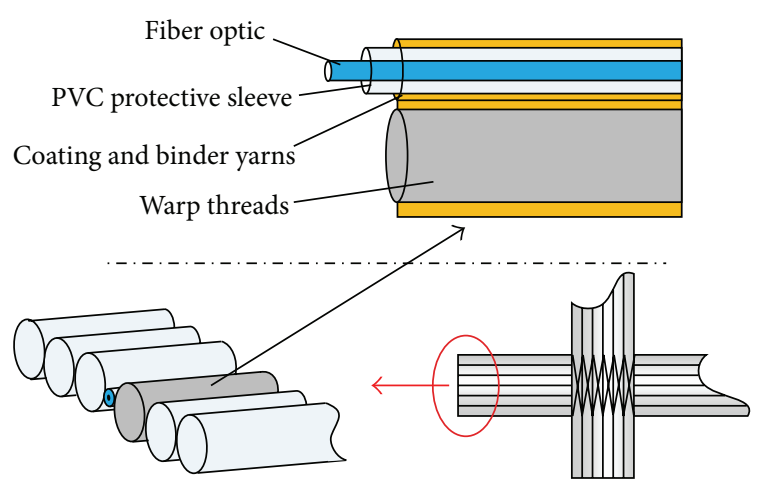

FIGURE 5: Schematic of strain transfer model.

and $0.4 \mathrm{~mm}$ displacement ( $1 \%$ elongation) was prescribed on the warps threads only. Simulation results are shown in Figure 6. The displacement results of fiber optic and one warp thread next to the fiber optic were displayed while other warp threads and the coating were hidden.

Comparison of the axial elongation of the two FBGs protected by PVC sleeve and nylon sleeve, respectively is shown in Figure 7. It is evident that with a tensile strain of $10000 \mu \varepsilon$ in the warps thread of geogrids, the fiber optic inside the PVC sleeve gets a strain of $9859 \mu \varepsilon$, which is about $98 \%$ of the actual strain, while fiber optic inside the nylon sleeve gets a strain of $9572 \mu \varepsilon$, which is about $95 \%$ of the applied strain. In contrast to nylon sleeve, the fiber optic with PVC sleeve is more suitable for smart geogrids as its higher strain transfer rate.

The strain transfer rates from geogrids to fiber optic with PVC sleeves in different elongations (i.e., $0.05 \%, 0.1 \%$, $0.3 \%, 0.5 \%, 1 \%, 1.5 \%, 2 \%$, and $3 \%$ ) are calculated, respectively (Figure 8). It indicates that the strain transfer rate will change with the applied elongation. Moreover, the minimum strain transfer rate (maximum strain loss) is approximately 0.983 when elongation is equal to $0.5 \%$. Although there is a slightly strain transfer loss in the smart geogrids, it is still acceptable for the strain measurement of geotechnical engineering application.

\section{Experiments and Discussions}

The sensing performance of proposed smart geogrids embedded with FBG is crucial to the application of smart geogrids for reinforcement as well as measurement. Thus, tensile experiments as well as temperature experiments were conducted to test the sensing characteristics. Warps knitted geogrids with mesh size of $40 * 40 \mathrm{~mm}$ were utilized as testing geogrids and FBGs with PVC sleeves were embedded in the warp threads.

4.1. Tensile Experiments. Two series of tensile experiments were carried out. The tensile displacement was applied by displacement calibrating bench, and the applied displacements were recorded by a digital displacement sensor with an accuracy of $0.01 \mathrm{~mm}$. The wavelengths of FBGs inside the geogrids were monitored by interrogator SM125, which has wavelength measurement accuracy of 1 pm from $1510 \mathrm{~nm}$ to $1590 \mathrm{~nm}$. The experimental setup is shown in Figure 9.

In the first stage, two ends of the testing geogrids embedded with one FBG with wavelength of $1540 \mathrm{~nm}$ were clamped on the calibrating bench. The length of testing geogrids was $400 \mathrm{~mm}$, and FBG was deployed in the middle of the testing geogrids. The geogrids were prestretched at the beginning of the each loading cycle to make sure the FBG inside geogrids was strained. The displacement was applied from $0 \mathrm{~mm}$ to $1 \mathrm{~mm}$, corresponding to $0 \sim 2500 \mu \varepsilon$, in an increment of approximately $0.1 \mathrm{~mm}(250 \mu \varepsilon)$ per step. After the loading cycle finished, the calibration bench was reset manually and the prestress was applied on the geogrids again. The same process was conducted three times, and the wavelength variation of embedded FBG with strain was obtained, as shown in Figure 10.

It can be seen in Figure 10 that the wavelength of FBG increases linearly with the elongation at the range of 0 $2500 \mu \varepsilon$. The relationship between Bragg wavelength and strain $\varepsilon$ of each time was fitted by Least-square algorithm, and the functions, respectively, are

$$
\begin{array}{ll}
\lambda_{B 1 \mathrm{st}}=9.10 \times 10^{-4} \cdot \varepsilon+1540.610 ; & R^{2}=0.9906 \\
\lambda_{B 2 \mathrm{nd}}=8.93 \times 10^{-4} \cdot \varepsilon+1540.722 ; & R^{2}=0.9994 \\
\lambda_{B 3 \mathrm{rd}}=8.84 \times 10^{-4} \cdot \varepsilon+1540.822 ; & R^{2}=0.9986 .
\end{array}
$$

The strain sensitivity each time is $0.910 \mathrm{pm} / \mu \varepsilon$, $0.893 \mathrm{pm} / \mu \varepsilon$, and $0.884 \mathrm{pm} / \mu \varepsilon$, respectively, and $R^{2}$ of each loading cycle is $0.9906,0.0094$, and 0.9986 , respectively, which indicate FBG inside the geogrids has good linearity. As expected, strain transfer losses exist although the value slightly larger than the simulated result. The strain sensitivity gradually decreases with testing time, which is partly due to the creep characteristics of PVC sleeve and geogrids which are fabricated by PET threads and polymer coating. In the first loading cycle, when Geogrids was stretched, the stress was transferred to the FBG and then led to wavelength shift. In the second loading cycle, due to the creep characteristics, the geogrids could not be recovered to its original length, and FBG was still stretched. If now same displacement was applied on the geogrids, FBG might suffer from less displacement which led to a smaller wavelength shift. As a result, the sensitivity decreased compared with that at the first time. The same situation might happen in the third loading cycle. Another reason for this phenomenon is that due to the precision limitation of the experiment setup, the calibrating bench need to be manually adjusted every time in order to prestretch the geogrids, which might induce errors. By averaging the sensitivity obtained by multiple tests, the deviation of can be minished. It should be noted that the deviation of original wavelength was attributed to the prestretching of the testing geogrids as the applied prestress was uneasy to be precisely controlled.

In the second series of experiments, geogrids embedded with four FBGs were tested to validate the response of FBGs in the same geogrids. The fiber optic cable was embedded in the centerline of warps threads and the positions of the four FBGs are as Figure 11. 


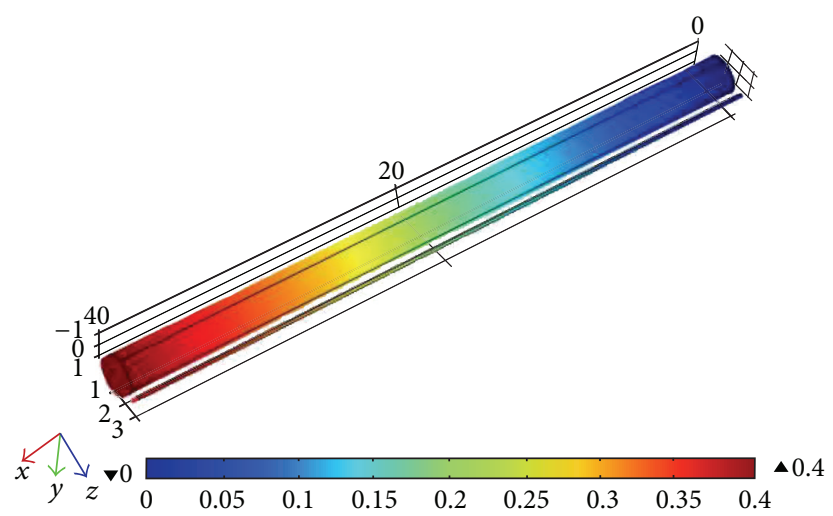

Figure 6: Displacement display of warps thread and fiber optic.

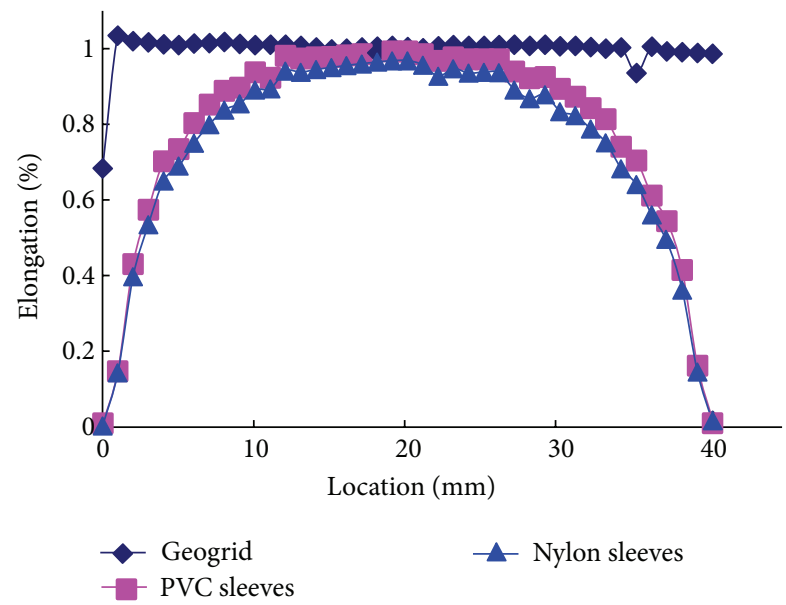

FIGURE 7: Simulation results of strain transfer.

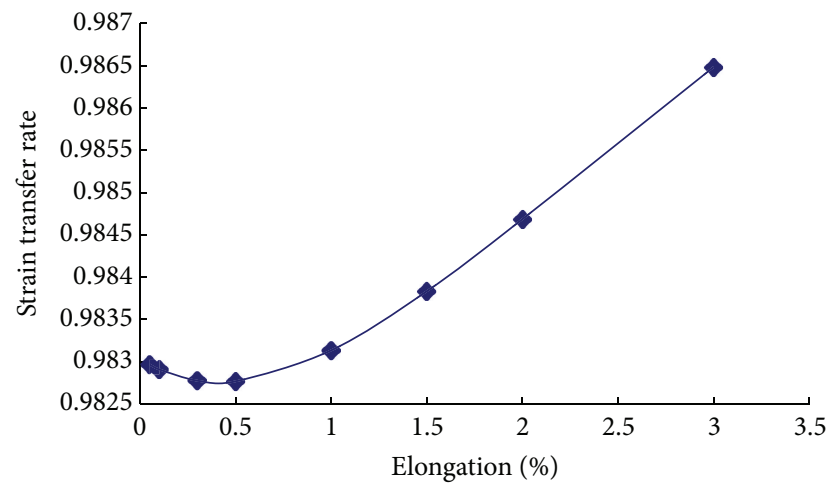

FIGURE 8: Variation of strain transfer rate with elongation.

The wavelengths of the four FBGs were $1536.187 \mathrm{~nm}$, $1542.732 \mathrm{~nm}, 1548.475 \mathrm{~nm}$, and $1552.721 \mathrm{~nm}$, respectively. The length of testing geogrids was $800 \mathrm{~mm}$, and displacement was applied from $0 \mathrm{~mm}$ to $1.6 \mathrm{~mm}$, corresponding to $0 \sim 2000 \mu \varepsilon$, in an increment of approximately $0.2 \mathrm{~mm}(250 \mu \varepsilon)$ per step. Experimental procedure was same as the first stage.

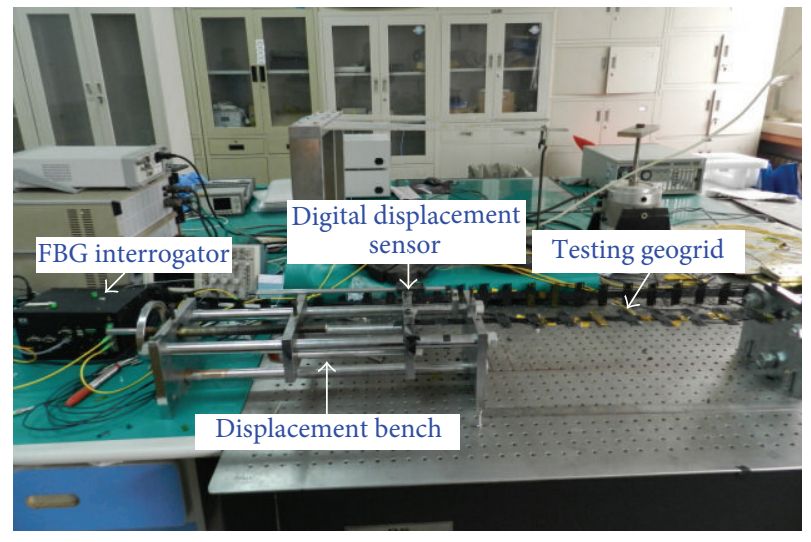

Figure 9: Tensile experiments setup.

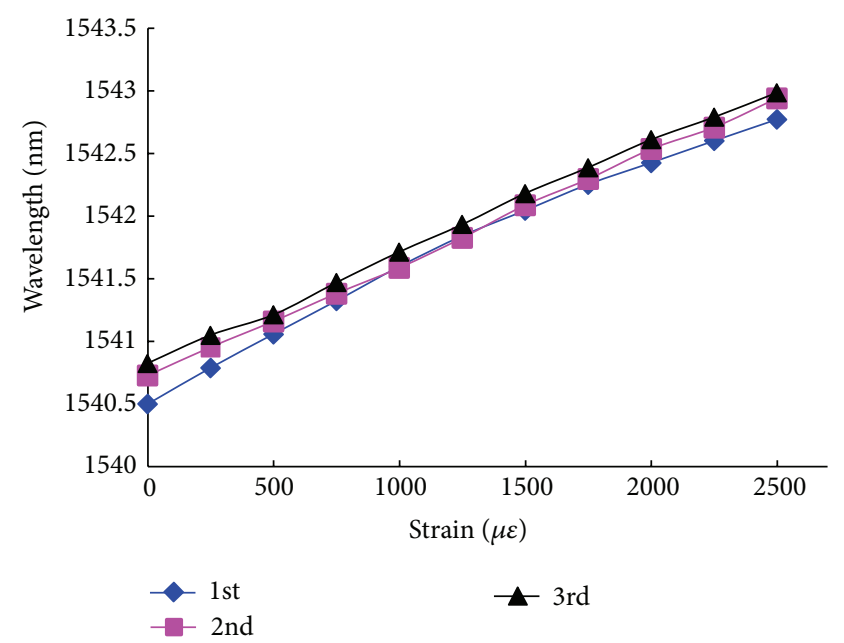

FIGURE 10: Results of tensile experiment.

To compare consistency of the four FBGs, the Bragg wavelength shifts $\Delta \lambda$ for each FBG are calculated by subtracting the original wavelength. Variation of $\Delta \lambda$ with strain $\varepsilon$ is shown in Figure 12. It can be seen that despite the slight fluctuations, the wavelengths of all FBGs shift linearly 


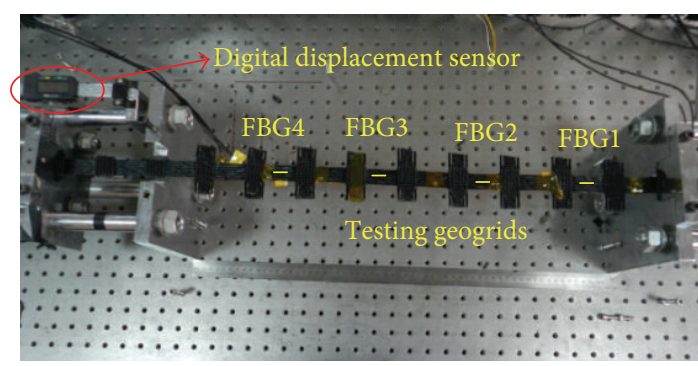

FIgUre 11: Position of four FBGs in the geogrids.

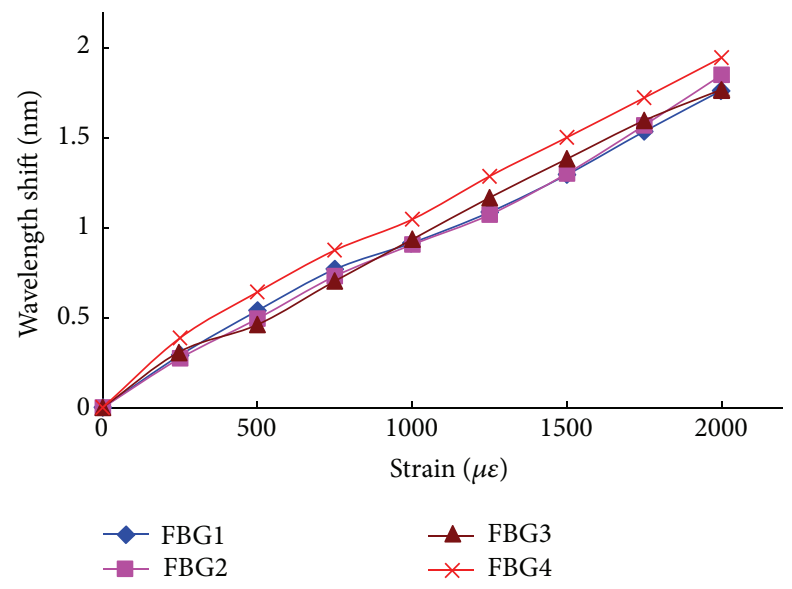

FIGURE 12: Results of tensile experiment.

with the applied strain. The relationship between Bragg wavelengths $\lambda$ and strain $\varepsilon$ of each FBG are

$$
\begin{array}{ll}
\lambda_{1}=8.42 \times 10^{-4} \cdot \varepsilon+1536.257 ; & R^{2}=0.994 ; \\
\lambda_{2}=8.84 \times 10^{-4} \cdot \varepsilon+1542.761 ; & R^{2}=0.996 ; \\
\lambda_{3}=8.83 \times 10^{-4} \cdot \varepsilon+1548.518 ; & R^{2}=0.998 ; \\
\lambda_{4}=9.29 \times 10^{-4} \cdot \varepsilon+1552.838 ; & R^{2}=0.993 .
\end{array}
$$

The results demonstrate that four FBGs inside the geogrids exhibit good linearity and consistency in the tensile test, and mean strain sensitivity of each FBG is $0.842 \mathrm{pm} / \mu \varepsilon$, $0.884 \mathrm{pm} / \mu \varepsilon, 0.883 \mathrm{pm} / \mu \varepsilon$, and $0.929 \mathrm{pm} / \mu \varepsilon$, respectively.

4.2. Temperature Experiments. As FBG is not only sensitive to axial strain but also to ambient temperature, it is necessary to perform experiments to evaluate the temperature performance of embedded FBG. A bared FBG and smart geogrids embedded with FBG were put into the temperature calibrating oven which provided constant temperature. The wavelength of FBG inside Geogrids was $1540 \mathrm{~nm}$, and so was the bared FBG. Temperature was varied from $25^{\circ} \mathrm{C}$ to $65^{\circ} \mathrm{C}$ with an increment of $10^{\circ} \mathrm{C}$ per step. Each step last for 1 hour for stabilization before recording the wavelength of the two FBGs.
Figure 13 shows the wavelength variation of the two FBGs with temperature. Within a temperature range $25^{\circ} \mathrm{C}-$ $65^{\circ} \mathrm{C}$, the wavelength of the $\mathrm{FBG}$ inside the geogrids increase with temperature, and its sensitivity is approximately $0.014 \mathrm{~nm} /{ }^{\circ} \mathrm{C}$. Compared with the bared FBG, whose sensitivity is $0.011 \mathrm{~nm} /{ }^{\circ} \mathrm{C}$, the embedded FBG exhibit larger sensitivity due to the influence of geogrids. Even though the result of embedded FBG did not exactly match with linear profile, the measurements were evenly distributed around it. Thus the temperature induced wavelength shifts of FBGs inside the geogrids could be compensated by extra FBG temperature sensors when using in practical application.

\section{Model Test}

During the construction of tunnel and other underground engineering, water inrushes occur frequently with disastrous consequences. For most of tunnels, the filling-type fissure widely exists in the rock as one kind of typical geological structure. To investigate the failure mechanism when water inrush happens in tunnel with the filling-type fissure, a geological model test was carried out to investigate water inrush process caused by filling body seepage failure in the practical engineering.

5.1. Model Test and Testing Procedure. A 3D visual water inrush model test system at a size of $3.0 \times 1.2 \times 2.7 \mathrm{~m}$ $(L \times W \times H)$ was set up to investigate tunnel's instability process during excavation (Figure 14). The model test system consisted of frame system, pressure loading system, and realtime data acquisition system. The frame system was made of steel combining with tempered glasses which were glued and sealed to realize $3 \mathrm{D}$ visualization. Excavation section was left in the front of tempered glasses, and one hole on each side of the frame system was left for sensor's wires. Similar materials for both surrounding rock as well as the filling body were developed and filled inside the frame system to simulate the properties of geological structure. The under width of fissure was $5 \mathrm{~cm}$ while the upper width was $10 \mathrm{~cm}$, and the length of fissure was $17 \mathrm{~cm}$. Angle of fissure with tunnel axis is $30^{\circ}$, and so the vertical direction, as shown in Figure 15. On the top of the similar materials a water storage section consisted of a permeable plate and pipes with holes was designed to provide water pressure for the system. Water was pumped from water tank to the water storage section. A water-resisting plate was covered and sealed to make the whole system water-tight. Jacks controlled by hydraulic pressure system were used for controllable load.

The real-time monitoring system was utilized to acquire information of key parameters such as: displacement, seepage, pressure, and strain. The sensors and geogrids were imbedded to the designed position when filling the similar materials. Three FBGs with the wavelength of $1536.187 \mathrm{~nm}$, $1542.732 \mathrm{~nm}$, and $1548.475 \mathrm{~nm}$ respectively were embedded in the geogrids, while a piece of FBG (1552.721 nm wavelength) embedded smart geogrids, which was protected to be strainindependent, was used for temperature compensation. Smart geogrids were placed at a groove behind the fissure to evaluate 


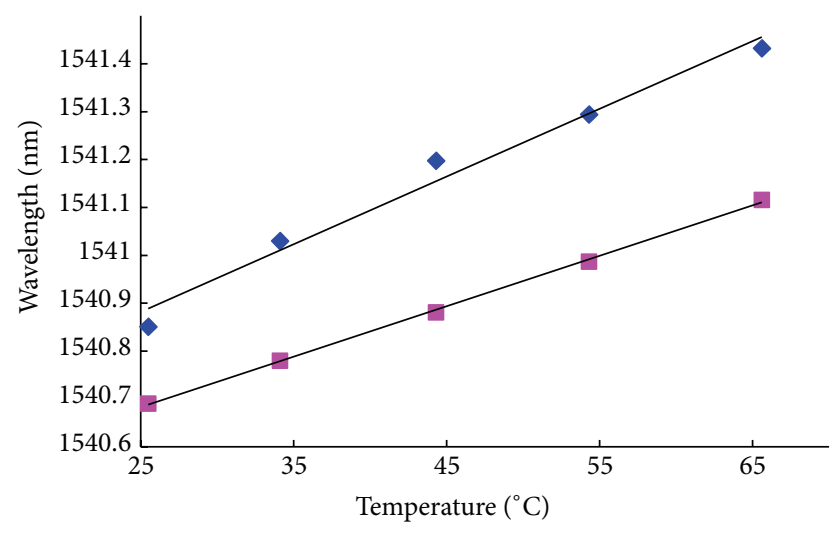

- FBG inside geogrid

- Bared FBG

Figure 13: Results of temperature experiment.

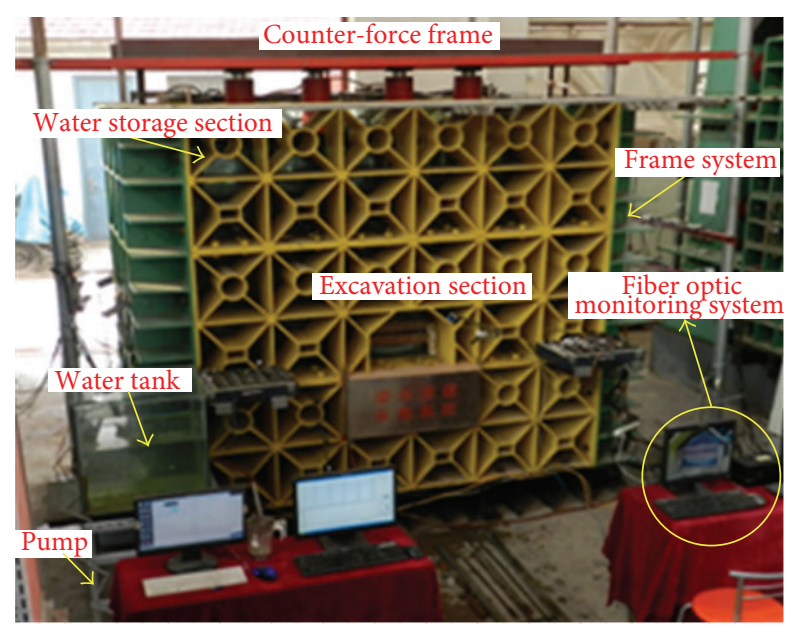

Figure 14: Photograph of the model test system.

strain variation during tunnel excavation. Each end of the geogrids was bound with a section of a PVC pipe which was inserted into the similar materials. Tense steel wires were bound and glued with PVC pipes at one end, and fixed on the steel frame at the other end. During the whole imbedding process, we kept tensing the geogrids to avoid bending. Similar materials were covered on tense geogrids and steel wires and then compacted to make sure no displacement on the boundary of the geogrids. Due to the water issue, the traditional strain gauges without waterproof were not suitable to be used in the model as the moisture might impact the strain reading. So FBG based strain sensor, which was developed by adhering 3 FBGs (vertical, horizontal, and slant $45^{\circ}$ ) on one surface of similar material block at a size of $3 \mathrm{~cm} \times$ $3 \mathrm{~cm} \times 3 \mathrm{~cm}$, was utilized as the traditional strain monitoring approach. The strain sensor, which was placed at one side of the fissure, was closed to the position of FBG2. The horizontal strain collected by this sensor was used to compare with strain collected by Geogrids. FBG interrogator was used to acquire wavelength signal and the data were saved in the computer.
The model test system and arrangement of Smart geogrids are shown in Figure 15.

The constant water pressure was loaded in the water storage section before excavation. The benching tunneling method was adopted for the excavation at a footage of $3 \mathrm{~cm}$ per step, while lower bench was $12 \mathrm{~cm}$ behind the upper bench during the excavation. The tunnel had a total length of $60 \mathrm{~cm}$, and the process consisted of 24 steps. Supporting was one step behind the excavation. The next step would begin after the tunnel was stable.

5.2. Results and Discussions. Figure 16 shows the strains measured by the smart geogrids and strain sensor during tunnel excavation. At the beginning of excavation (Step 1Step 8), the variation of measured strains increased gradually as surrounding rock and filling body in the fissure were relatively steady. Due to the water pressure, filling body which consisted of similar materials with large particles and low strength was compressed. As a result, the measured strains showed a slightly increase from Step 2 to Step 4 . At Step 5 and Step 6, the strains decreased probably because water which was originally reserved in the filling body was permeated into tunnel through some cracks. This phenomenon was also captured by the strain sensor. From Step 9 to Step 13, as tunnel face went through the fissure, the stress of surrounding rock and filling body was released, which led to a rapid increase of the strains. At this stage, the strain variation measured by the strain sensor was larger than the one measured by FBG2 of Geogrids, which is possibly because the strain sensor was placed more closed to fissure. After Step 13, the tunnel face had gone through the fissure, but the strains kept increasing until Step 16. Thereafter, the strains were gradually stabilized. Although the strains measured by Geogrids were slightly smaller than that measured by strain sensor, the tendency agreed very well. The strain of FBG2 which was close to arch crown was larger than strains sensed by the other two FBGs during the whole excavation process. This phenomenon indicated that the deformation of arch crown was larger.

During the total process of model test, the smart geogrids exhibited desirable performance. The test results demonstrate that proposed smart geogrids embedded with FBG can be an effective and promising geosynthetics method for the realtime monitoring of strains for geological model test and other geotechnical engineering related applications.

\section{Conclusion}

This paper develops smart geogrids embedded within FBGs for reinforcement and measurement of geotechnical engineering applications as well as geotechnical model tests. The fabricating process of smart geogrids has been introduced in detail. The strain distribution within the warps threads of geogrids' ribs is investigated by finite element simulation. To protect the fragile fiber optic and minimize strain transfer error, the strain transfer characteristics of geogrids embedded with fiber optic have been analyzed base on the simulation results, which indicate that protective sleeve made by PVC have better strain transfer performance than that made by 


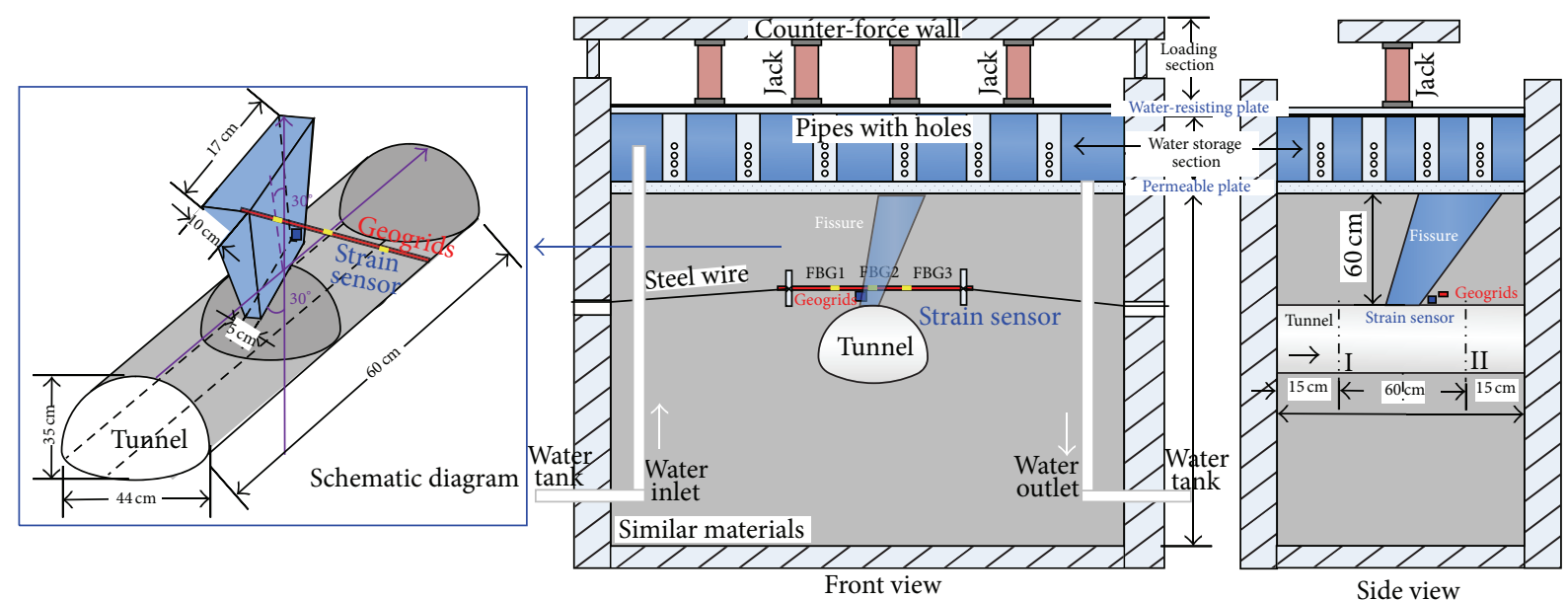

FIGURE 15: The model test system and arrangement of Smart geogrids.

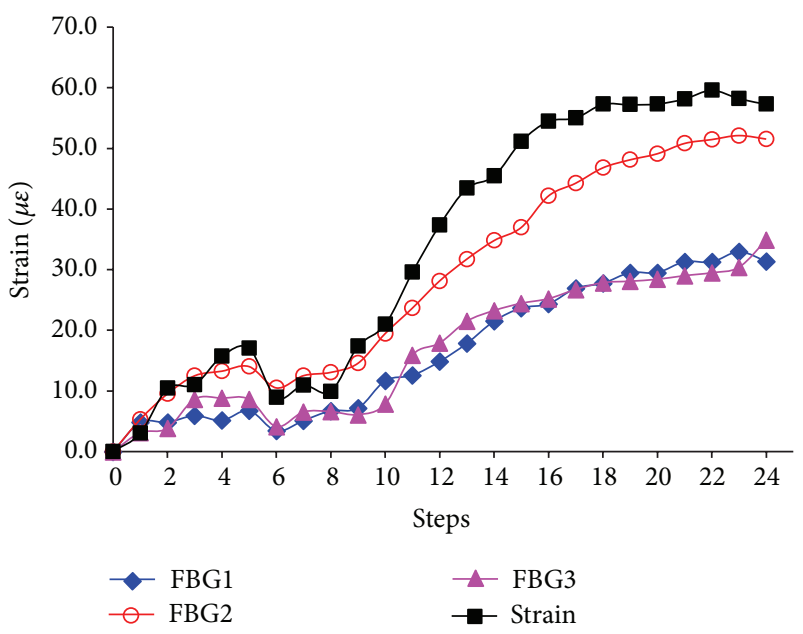

FIGURE 16: Strain results of Geogrids and strain sensor.

nylon, and the minimum strain transfer rate of PVC sleeves is approximately 0.983 when elongation is $0.5 \%$ at a the range of $0 \% \sim 3 \%$. A series of experiments, including tensile experiments and temperature experiments, have been conducted to verify the performance of proposed smart geogrids. The results of tensile experiment demonstrate that the wavelength of FBGs embedded within geogrids varies linearly with the tensile displacement, and the proposed smart geogrids also exhibit good consistency. Temperature experiment show that the temperature sensitivity of FBG integrated with geogrids is slightly higher than that of the normal FBG, and the temperature induced wavelength shifts can be compensated by an extra FBG temperature sensors. Finally, the designed smart geogrids are used in a geotechnical model test to monitor strains during the tunnel excavation, and results indicate that proposed smart geogrids embedded with FBGs can be usd effectively to measure strains for geological engineering related applications.

\section{Conflict of Interests}

The authors declare that there is no conflict of interests regarding the publication of this paper.

\section{Acknowledgments}

The research of this paper is supported by the National Science Foundation of China (41472260 and 41202206) and the Science Project of Suzhou City (SYG201306). The authors also express their appreciation to TAIAN Road Engineering Materials co. Ltd.

\section{References}

[1] M. A. El Sawwaf, "Behavior of strip footing on geogridreinforced sand over a soft clay slope," Geotextiles and Geomembranes, vol. 25, no. 1, pp. 50-60, 2007.

[2] K. Kazimierowicz-Frankowska, "Influence of geosynthetic reinforcement on the load-settlement characteristics of two-layer subgrade," Geotextiles and Geomembranes, vol. 25, no. 6, pp. 366-376, 2007.

[3] G. M. Latha and V. S. Murthy, "Effects of reinforcement form on the behavior of geosynthetic reinforced sand," Geotextiles and Geomembranes, vol. 25, no. 1, pp. 23-32, 2007.

[4] H. Zhou and X. Wen, "Model studies on geogrid- or geocellreinforced sand cushion on soft soil," Geotextiles and Geomembranes, vol. 26, no. 3, pp. 231-238, 2008.

[5] K. S. C. Kuang, C. Y. Tan, S. H. Chew, and S. T. Quek, "Monitoring of large strains in submerged geotextile tubes using plastic optical fibre sensors," Sensors and Actuators A: Physical, vol. 167, no. 2, pp. 338-346, 2011.

[6] R. K. Rowe and C. T. Gnanendran, "Geotextile strain in a full scale reinforced test embankment," Geotextiles and Geomembranes, vol. 13, no. 12, pp. 781-806, 1994.

[7] R. K. Rowe and B. L. J. Mylleville, "A geogrid reinforced embankment on peat over organic silt: a case history," Canadian Geotechnical Journal, vol. 33, no. 1, pp. 106-122, 1996. 
[8] C. T. Gnanendran and A. P. S. Selvadurai, "Strain measurement and interpretation of stabilising force in geogrid reinforced," Geotextiles and Geomembranes, vol. 19, no. 3, pp. 177-194, 2001.

[9] B. V. S. Viswanadham and D. König, "Studies on scaling and instrumentation of a geogrid," Geotextiles and Geomembranes, vol. 22, no. 5, pp. 307-328, 2004.

[10] B.-J. Wang, K. Li, B. Shi, and G.-Q. Wei, “Test on application of distributed fiber optic sensing technique into soil slope monitoring," Landslides, vol. 6, no. 1, pp. 61-68, 2009.

[11] H.-H. Zhu, J.-H. Yin, A. T. Yeung, and W. Jin, "Field pullout testing and performance evaluation of GFRP soil nails," Journal of Geotechnical and Geoenvironmental Engineering, vol. 137, no. 7, pp. 633-642, 2011.

[12] W. R. Habel and K. Krebber, "Fiber-optic sensor applications in civil and geotechnical engineering," Photonic Sensors, vol. 1, no. 3, pp. 268-280, 2011.

[13] H.-H. Zhu, A. N. L. Ho, J.-H. Yin, H. W. Sun, H.-F. Pei, and C.-Y. Hong, "An optical fibre monitoring system for evaluating the performance of a soil nailed slope," Smart Structures and Systems, vol. 9, no. 5, pp. 393-410, 2012.

[14] C.-C. Zhang, H.-H. Zhu, Q. Xu, B. Shi, and G. Mei, "Timedependent pullout behavior of GFRP soil nail in sand," Canadian Geotechnical Journal, vol. 51, pp. 1-10, 2014.

[15] X. Weng, H.-H. Zhu, J. Chen, D. Liang, B. Shi, and C.-C. Zhang, "Experimental investigation of pavement behavior after embankment widening using a fiber optic sensor network," Structural Health Monitoring, vol. 14, no. 1, pp. 46-56, 2014.

[16] C.-C. Zhang, H.-H. Zhu, B. Shi, F.-D. Wu, and J.-H. Yin, "Experimental investigation of pullout behavior of fiber-reinforced polymer reinforcements in sand," Journal of Composites for Construction, Article ID 04014062, 2014.

[17] H.-H. Zhu, J.-H. Yin, L. Zhang, W. Jin, and J.-H. Dong, "Monitoring internal displacements of a model dam using FBG sensing bars," Advances in Structural Engineering, vol. 13, no. 2, pp. 249-261, 2010.

[18] H.-H. Zhu, J.-H. Yin, J.-H. Dong, and L. Zhang, "Physical modelling of sliding failure of concrete gravity dam under overloading condition," Geomechanics and Engineering, vol. 2, no. 2, pp. 89-106, 2010.

[19] H.-H. Zhu, B. Shi, J.-F. Yan, J. Zhang, C.-C. Zhang, and B.J. Wang, "Fiber Bragg grating-based performance monitoring of a slope model subjected to seepage," Smart Materials and Structures, vol. 23, no. 9, Article ID 095027, 2014.

[20] C.-C. Zhang, H.-H. Zhu, B. Shi, and J.-K. She, "Interfacial characterization of soil-embedded optical fiber for ground deformation measurement," Smart Materials and Structures, vol. 23, no. 9, Article ID 095022, 2014.

[21] H.-H. Zhu, B. Shi, J. Zhang, J.-F. Yan, and C.-C. Zhang, "Distributed fiber optic monitoring and stability analysis of a model slope under surcharge loading," Journal of Mountain Science, vol. 11, no. 4, pp. 979-989, 2014.

[22] N. Nöther, A. Wosniok, K. Krebber, and E. Thiele, "A distributed fiber optic sensor system for dike monitoring using Brillouin optical frequency domain analysis," in Optical Sensors, vol. 7003 of Proceedings of SPIE, pp. 69330T-1-69330T-9, April 2008.

[23] E. Thiele, R. Helbig, H. Erth et al., "Dike monitoring," in Proceedings of the 4th International Symposium on Flood Defense, pp. 19-1-19-7, 2008.
[24] K. Krebber, S. Liehr, and J. Witt, "Smart technical textiles based on fiber optic sensors," in 22nd International Conference on Optical Fiber Sensors (OFS '12), vol. 8421 of Proceedings of SPIE, pp. 84212A-1-84212A-10, October 2012.

[25] C. Crunelle, M. Wuilpart, C. Caucheteur, and P. Mégret, "Original interrogation system for quasi-distributed FBG-based temperature sensor with fast demodulation technique," Sensors and Actuators A: Physical, vol. 150, no. 2, pp. 192-198, 2009. 

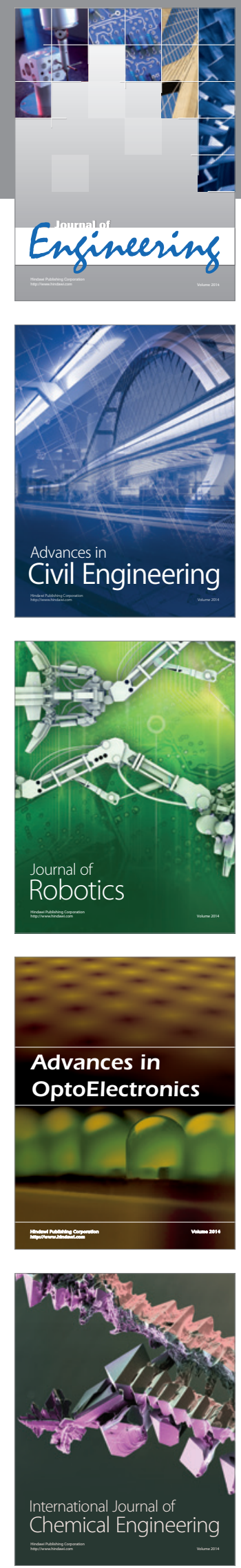

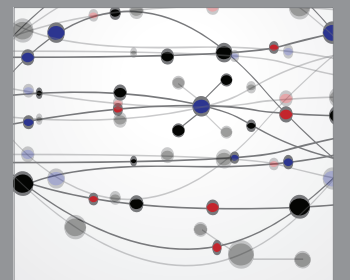

The Scientific World Journal
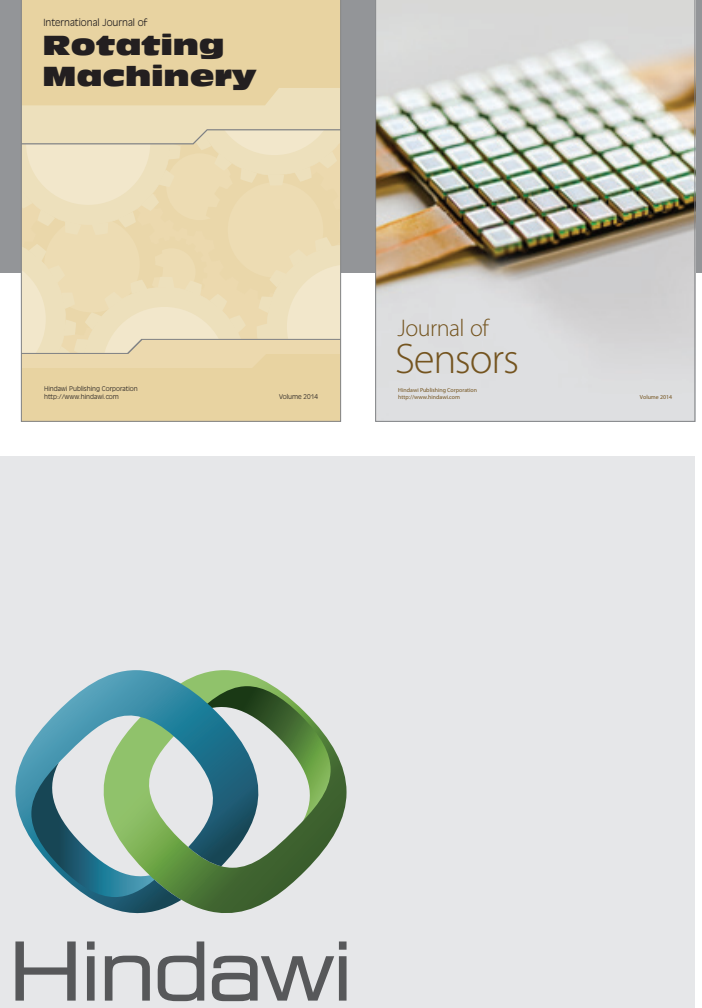

Submit your manuscripts at http://www.hindawi.com
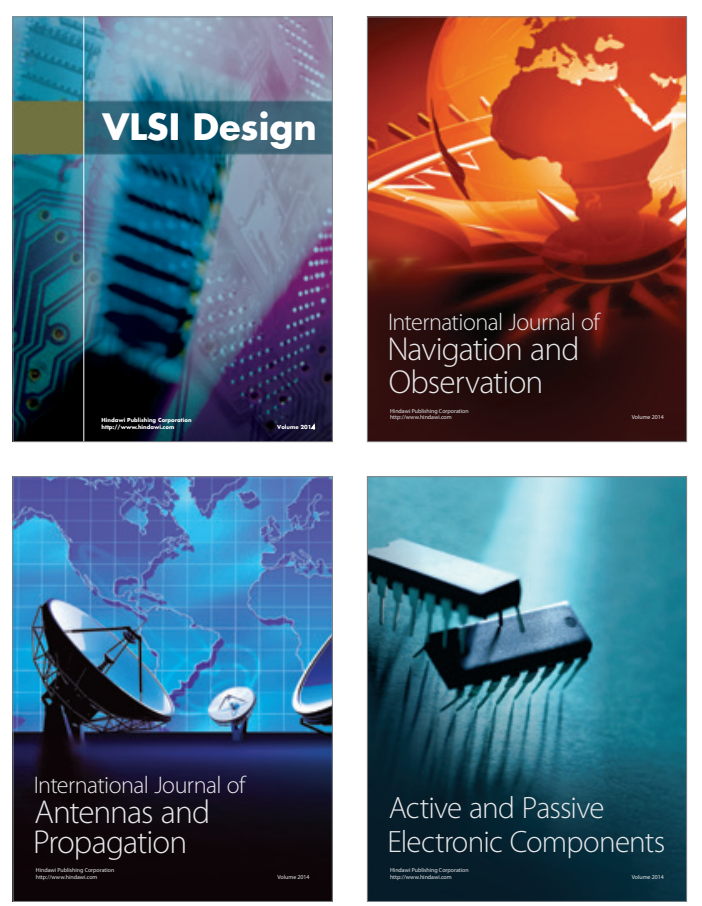
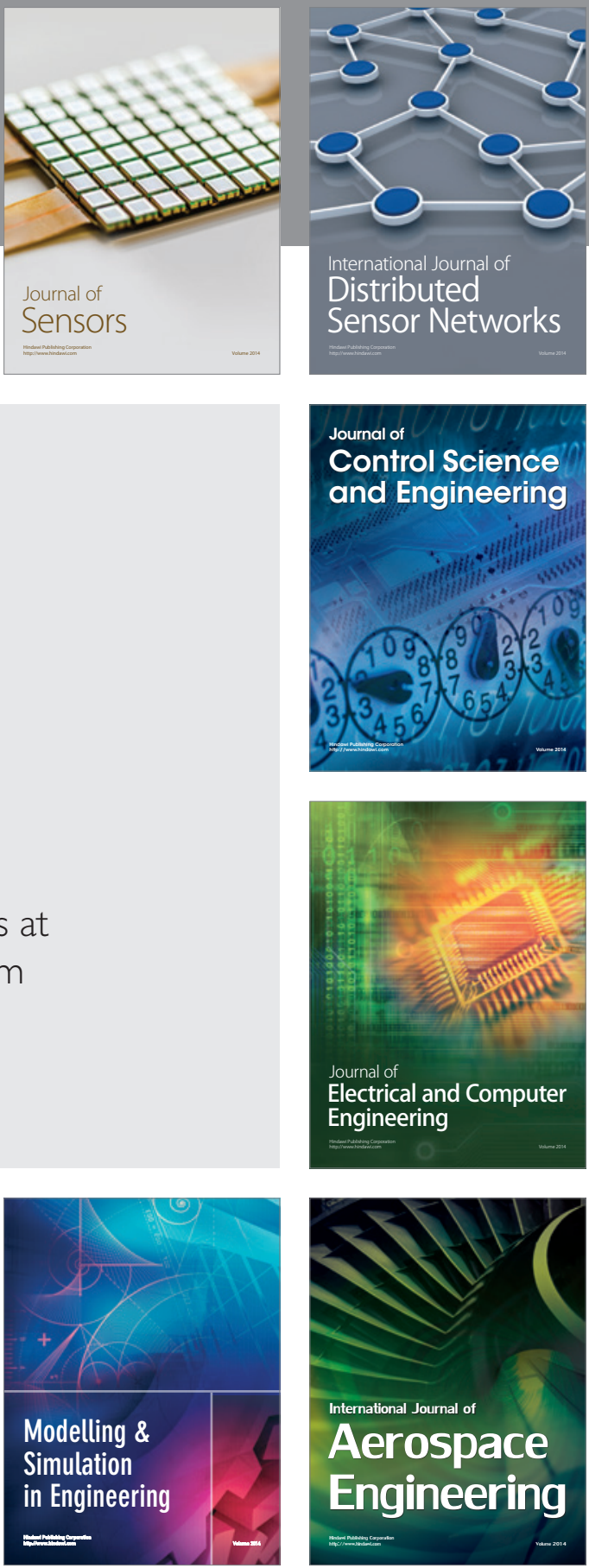

Journal of

Control Science

and Engineering
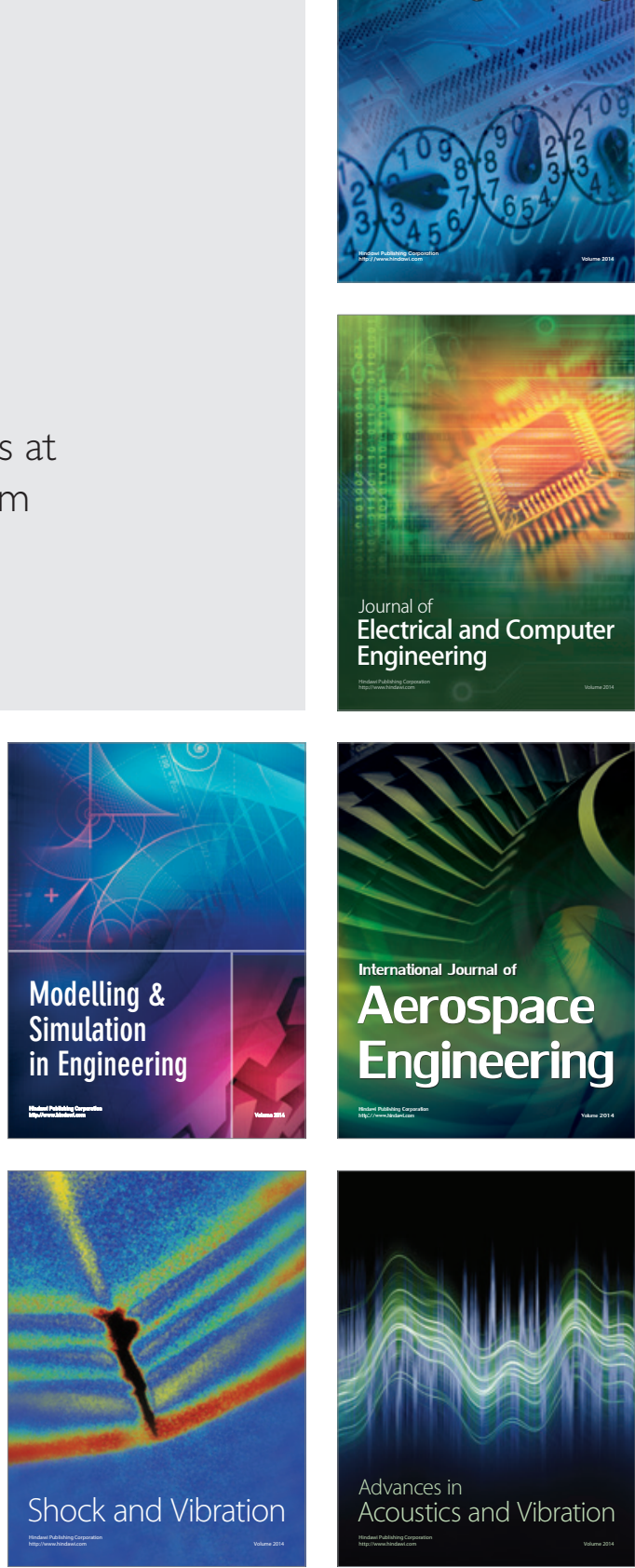